\title{
Hipermetabolismo en el paciente quemado
}

Hypermetabolism in the burned patient

Hipermetabolismo nos pacientes queimados

Marco Antonio Garnica Escamilla, ${ }^{*}$ Jordana Lemus Sandoval, ${ }^{\ddagger}$ Bricia Noemí Ramírez Martínez,

Elvira Alejandra Tamez Coyotzin, ${ }^{*}$ Oscar Miguel Marín Landa*

\section{RESUMEN}

La lesión por quemadura se denomina con frecuencia la forma más grave de trauma en relación con la elevada respuesta metabólica que genera debido a que puede perpetuarse y permanecer elevada incluso hasta tres años después de que la lesión se ha resuelto.

Las alteraciones en el metabolismo son complejas debido a los diversos mecanismos involucrados (hormonales, inflamatorios, metabólicos y genéticos). Existen también en la actualidad diversos mecanismos implementados con la finalidad de tratar de frenar esta respuesta hipermetabólica. La finalidad de este artículo es dar a conocer al personal médico cuáles son las principales causas del hipermetabolismo y las estrategias de manejo que existen en el tratamiento del quemado grave.

Palabras clave: Hipermetabolismo, nutrición, paciente quemado.

\section{ABSTRACT}

Burn injury is often referred to as the most severe form of trauma in relation to the high metabolic response it generates because it can be perpetuated and remain elevated even up three years after the injury has resolved.

Metabolic alterations are complex due to various mechanisms involved (metabolic, hormonal, inflammatory and genetic). Currently there are several mechanisms implemented in order to try to stop this hypermetabolic response. The purpose of this article is to inform medical personnel, which are the main causes of hypermetabolism and management strategies that exist in the treatment of severe burn.

Keywords: Hypermetabolism, nutrition, burned patient.

\section{RESUMO}

A lesão por queimadura é freqüentemente referida como a forma mais grave de trauma em relação à alta resposta metabólica que gera, pois pode perpetuarse e permanecer elevada até três anos após a resolução da lesão.

As alterações do metabolismo são complexas devido aos vários mecanismos envolvidos (hormonais, inflamatórios, metabólicos e genéticos). Existem também atualmente vários mecanismos implementados a fim de tentar parar esta resposta hipermetabólica.

O objetivo deste artigo é dar a conhecer à equipe médica, quais são as principais causas do hipermetabolismo e as estratégias de manejo que existem no tratamento de queimaduras graves.

Palavras-chave: Hipermetabolismo, nutrição, paciente queimado.

\section{INTRODUCCIÓN}

La lesión inducida por quemadura genera una grave respuesta inmunometabólica y un hipercatabolismo acentuado. La duración de la respuesta catabólica en las quemaduras puede permanecer elevada incluso años después de la lesión. Existen otros escenarios clínicos, por ejemplo, el paciente traumatizado o la sepsis, que también generan hipermetabolismo, pero el grado de

\footnotetext{
* Centro Nacional de Investigación y Atención de Quemados. Instituto Nacional de Rehabilitación, «Luis Guillermo Ibarra Ibarra».

‡ Unidad de Cuidados Intensivos, Hospital General «Dr. Manuel Gea González».

Recibido: 29/01/2020. Aceptado: 24/02/2020

Citar como: Garnica EMA, Lemus SJ, Ramírez MBN, Tamez CEA, Marín LOM. Hipermetabolismo en el paciente quemado. Med Crit. 2021;35(4):194-199. https://dx.doi. org/10.35366/101158

www.medigraphic.com/medicinacritica
}

respuesta es menor comparado con el paciente quemado. Esto implica que no existe una condición clínica que iguale la respuesta metabólica que genera el paciente con quemaduras graves. Este hipermetabolismo genera un aumento en el consumo de $\mathrm{O}_{2}$ de todo el organismo, representando un componente significativo del gasto total de energía, donde hasta $45 \%$ de esta respuesta hipermetabólica es atribuible a la producción de calor. ${ }^{1}$

Como consecuencia, el hipermetabolismo induce a un vasto catabolismo que se asocia con la degradación de proteínas no sólo en los músculos, sino en casi todos los órganos y sistemas, lo que puede acentuar la disfunción multiorgánica presente, impactando directamente en la supervivencia del paciente quemado. La respuesta hipermetabólica posterior a la quemadura es extremadamente compleja y es muy probable que esté activa y sea sostenida por la inflamación y la liberación hormonal inducida por este estado de estrés. La causa de esta respuesta no está completamente definida; sin embargo, se han visto involucrados la expresión aumentada y prolongada de catecolaminas, glucocorticoides, glucagón y dopamina, conduciendo a un estado catabólico profundo. ${ }^{2}$

Los cambios metabólicos posteriores a la lesión por quemadura ocurren en dos patrones distintos de regulación metabólica: la fase EBB generalmente ocurre dentro de las 48 horas postquemadura y se caracteriza por disminución del gasto cardiaco, menor consumo de oxígeno y menor tasa metabólica. La respuesta metabólica aumenta gradualmente dentro de los primeros cinco días posteriores a la lesión a una fase de meseta: la fase FLOW se caracteriza por una circulación hiperdinámica, aumentos de la temperatura corporal, consumo de oxígeno y glucosa, producción de $\mathrm{CO}_{2}$, gluconeogénesis, proteólisis, lipólisis. ${ }^{3}$

El catabolismo crónico del músculo esquelético y el desgaste muscular resultante es patognomónico del paciente con quemaduras severas. Esta erosión de la masa corporal magra puede retrasar la curación y contribuir significativamente a la morbilidad a largo plazo de los pacientes.

El musculoesquelético cumple dos funciones esenciales, una contráctil para la locomoción y el mantenimiento de la postura y una función metabólica como reservorio de proteína del cuerpo (proteínas miofibrilares). Una vez agotadas las reservas hepáticas de glucógeno, 
se activa la degradación de proteínas musculares para proporcionar aminoácidos a la gluconeogénesis. Estos aminoácidos también pueden oxidarse para la producción de energía. Se ha demostrado que la atrofia del musculoesquelético es un factor de riesgo independiente que predice una disminución de la supervivencia en muchas enfermedades.

Las lesiones por quemadura y la capacidad de afectar la masa muscular está mediada por vías de señalización endocrinas, paracrinas y autocrinas, que influyen en el tamaño muscular mediante la regulación de las tasas de síntesis y degradación de proteína. La principal de estas señales es la vía de la insulina/IGF-I (Factor de crecimiento similar a la insulina), que promueve el crecimiento muscular. Otras vías anabólicas incluyen aquellas activadas por andrógenos y por proteínas morfogenéticas óseas. Las vías de señalización catabólica son numerosas, consistentes con la multitud de trastornos que pueden causar desgaste muscular. La miostatina, un miembro de la familia TGF- $\beta$ (factor de crecimiento transformador-beta) se produce en el musculoesquelético y puede promover la atrofia muscular de forma paracrina y autocrina. Los glucocorticoides son una señal catabólica común. Muchas citoquinas inflamatorias también activan vías de señalización en el músculo para promover el catabolismo. Se reconoce que tanto la proteólisis autofágica/lisosómica como el sistema ubiquitina proteasoma (UPS) juegan un papel importante en la degradación de proteínas. ${ }^{4}$

El sistema de ubiquitinación de proteínas fue descrito por el grupo del Dr. Ciechanover, logro que fuera merecedor de un premio Nobel. Este grupo caracterizó la función de la ubiquitina como una marca para la subsecuente degradación de las proteínas en el proteasoma, en especial para proteínas de vida media corta. Sin embargo, se ha descubierto que la ubiquitinación tiene otras funciones: participa en la regulación de vías de señalización intercelular, involucrándose en funciones tan diversas como el control de la apoptosis, la autofagia, el ciclo celular, la regulación transcripcional y la reparación del ADN. ${ }^{5}$

Durante la respuesta hipermetabólica se ha observado mayor liberación de insulina con niveles de glucosa en plasma marcadamente elevados, lo que indica el desarrollo de resistencia a la insulina. ${ }^{6}$ Además, la lipólisis aumenta sustancialmente y aumenta el número de ácidos grasos libres y triglicéridos. ${ }^{7}$ Se ha demostrado que los niveles totales de cortisol urinario, las citocinas séricas, las catecolaminas séricas y los requerimientos de energía basal se mantienen significativamente elevados durante hasta tres años posterior a la lesión, acompañados por un metabolismo alterado de la glucosa y sensibilidad a la insulina. Estos resultados indican la importancia del seguimiento a largo plazo y tratamiento de pacientes gravemente quemados.
El control deficiente de la glucosa puede ser provocado por una pérdida de sensibilidad a la insulina hepática (central) y del musculoesquelético (periférico); por un lado, la capacidad disminuida de la insulina para suprimir la producción de glucosa hepática (resistencia central) y la disminuida capacidad para la utilización de la glucosa en el músculo esquelético (resistencia periférica). Por lo tanto, parece que los pacientes con quemaduras experimentan un «doble golpe» donde la sensibilidad a la insulina tanto central como periférica disminuye después de la quemadura, lo que da como resultado un control deficiente de la glucosa. ${ }^{8}$ La hiperglucemia es perjudicial y se asocia con aumento del número de infecciones, mayor incidencia de sepsis, catabolismo e hipermetabolismo significativamente mayores y, lo que es más importante, un incremento de la mortalidad posterior a la quemadura. ${ }^{9}$

La otra ruta metabólica que se altera significativamente durante la respuesta hipermetabólica es el metabolismo de los lípidos, que puede estar relacionado con cambios en la resistencia a la insulina. Una de las principales alteraciones posteriores a la lesión es un aumento significativo de la lipólisis atribuible al aumento de los niveles de catecolaminas.

La lipólisis consiste en la degradación (hidrólisis) de triacilglicerol en ácidos grasos libres (AGL) y glicerol. Los AGL deterioran la captación de glucosa estimulada por insulina e inducen mayor resistencia a la insulina a través de la inhibición de la actividad de transporte de glucosa.

El aumento de los triglicéridos y de los ácidos grasos libres conduce a la infiltración grasa de órganos vitales, especialmente el hígado. En consecuencia, el hígado graso es muy común después de la quemadura y se asocia con un aumento de la morbilidad clínica, así como alteraciones metabólicas. El mecanismo por el cual los lípidos inducen la resistencia a la insulina no está completamente definido. El tejido adiposo fue ignorado como un contribuyente a la respuesta hipermetabólica durante mucho tiempo, pero es cada vez más evidente que juega un papel muy importante en la mediación no sólo de respuestas metabólicas, sino también inflamatorias. ${ }^{10}$

\section{Manejo de la respuesta hipermetabólica}

Manejo ambiental: la piel aísla al cuerpo y juega un papel central en la termorregulación. En consecuencia, la destrucción de esta barrera significa que los sobrevivientes de quemaduras necesitan producir más calor para mantener la neutralidad térmica. El aumento de la temperatura ambiente en las habitaciones de los pacientes y el uso de vendajes oclusivos limitan la respuesta hipermetabólica a las quemaduras. La importancia de la escisión inmediata, el cierre temprano o la colocación de injertos (en los casos que esto se 
requiera) de las lesiones por quemadura son una piedra angular del tratamiento, que ha demostrado reducir incidencia de sepsis y mortalidad. La termogénesis mitocondrial sigue siendo un componente significativo del hipermetabolismo inducido por quemaduras, es por ello que las nuevas tecnologías para cobertura de heridas tales como productos sintéticos para la piel, terapias farmacológicas y las estrategias medioambientales deberían explorarse como un medio para mitigar el hipermetabolismo después de una lesión por quemadura. ${ }^{1}$

Manejo nutricional: el paciente quemado crítico se caracteriza por el desarrollo precoz de un estado de hipermetabolismo e hipercatabolismo severo, con un aumento de la tasa metabólica basal (hasta $80 \%$ por encima de los valores normales) con importantes alteraciones de la función mitocondrial. El daño térmico es una importante causa de pérdida de macronutrientes (proteínas) y micronutrientes (elementos traza y vitaminas) a través de las áreas quemadas. La intensa respuesta hipermetabólica e hipercatabólica genera el desarrollo de malnutrición aguda, sarcopenia secundaria y debilidad muscular adquirida. Por otra parte, el balance negativo de micronutrientes antioxidantes durante el daño térmico favorece el desarrollo de estrés oxidativo. En tal sentido, la implementación de una terapia nutricional adecuada, precoz e individualizada ha demostrado ser capaz de mejorar los resultados clínicos, en particular disminuyendo la incidencia de complicaciones infecciosas, la estancia hospitalaria y acelerando el proceso de cicatrización de heridas. ${ }^{11}$

La vía de nutrición enteral (NE) es de elección en el paciente quemado crítico. La NE precoz en las primeras seis a 12 horas es segura y capaz de modular la respuesta hipermetabólica, disminuyendo de modo significativo los niveles de catecolaminas, cortisol y glucagón, con un aumento concomitante de la producción de inmunoglobulinas. Los requerimientos nutricionales del paciente quemado grave son elevados debido al hipermetabolismo e hipercatabolismo severos. El requerimiento energético debe ser estimado de forma diaria e individualizada, preferiblemente mediante el uso de calorimetría indirecta, la cual actualmente es considerada el estándar de oro. ${ }^{12,13}$

Con relación al uso de las ecuaciones predictoras, la ecuación de Toronto parece ser la más adecuada, ya que considera la variabilidad de los requerimientos energéticos a medida que evoluciona la enfermedad, en tanto que para niños quemados críticos la fórmula de Schofield ha demostrado ser de elección (Tabla 1). ${ }^{14}$

Los hidratos de carbono $(\mathrm{HC})$ deben ser aportados a una dosis de 4-5 g/kg/día, no debiéndose superar un aporte de calorías de 1,400-1,500 kcal/día como HC. La proporción de grasas debe estar en torno a 15-18\%, no superando $20-30 \%$ del aporte calórico total no-proteico (1.0-1.5 g/kg/día). El requerimiento proteico debe ser no menor al $20-25 \%$ del aporte calórico total (2.0-2.5 $\mathrm{g} / \mathrm{k} / \mathrm{día}){ }^{15}$

Por el momento, no existe una nutrición ideal para los pacientes quemados y no existe un estándar de oro. Se considera que la principal fuente de energía deben ser los hidratos de carbono y los aminoácidos, evitando así que las proteínas se oxiden para obtener energía, lo que permite que la piel y los órganos usen la proteína de manera eficaz.

La glutamina, un aminoácido condicionalmente esencial en la enfermedad crítica, ha sido un tópico de debate en los últimos años. La glutamina se agota rápidamente después de una quemadura; sin embargo, este agotamiento se produce principalmente de manera intracelular y es muy difícil administrar glutamina de forma efectiva a las células. La mayor evidencia que apoya la suplementación de glutamina en pacientes quemados proviene de estudios que han demostrado disminución de la incidencia de infecciones, días de estancia intrahospitalaria y mortalidad, aunque su uso aún sigue siendo controversial.

\section{Micronutrientes}

El metabolismo de numerosos micronutrientes (vitaminas y elementos traza) es benéfico e importante para la cicatrización de las heridas y la función inmunológica en los pacientes quemados. La noción de suplementar vitaminas en pacientes quemados surge por la evidencia de niveles séricos bajos de algunas vitaminas claves como la A, C, y D. La vitamina $C$ es un nutriente crítico vital, ya que promueve la síntesis y reticulación de colágeno, así como la neutralización de radicales libres presentes durante la lesión térmica. Tiene un reconocido efecto protector endotelial mediante la prevención de la desfosforilación, con lo cual disminuye la hiperpermeabilidad capilar y mejora el flujo en la microcirculación. ${ }^{11}$

Tabla 1: Ecuaciones predictivas para el cálculo de necesidades energéticas en el paciente quemado crítico.

Ecuación de Toronto (resultado se aproxima a calorimetría indirecta)

REE $=\left(-4343+[10.5 \times \%\right.$ SCQ $]+[0.23 \times$ ingesta calórica $]+[0.84 \times$ Harris Benedict $]+\left[114 \times\right.$ temperatura rectal en $\left.{ }^{\circ} \mathrm{C}\right]-[4.5 \times$ día posquemadura $\left.]\right)$

Ecuación de Harris-Benedict (el resultado debe multiplicarse por el factor de estrés [1.2 a 2.0])

Hombres: $66.437+(5.0033 \times$ altura $[\mathrm{cm}])+(13.7516 \times$ peso $[\mathrm{kg}])$-edad [años] $)$

Mujeres: $655.0955+(1.8496 \times$ altura $[\mathrm{cm}])+(9.5634 \times$ peso $[\mathrm{kg}])-(4.6756 \times$ edad [años] $)$

$\mathrm{REE}=$ requerimientos energéticos estimados; $\mathrm{SCQ}=$ superficie corporal total quemada. 
Tabla 2: Nutrición en paciente crítico con lesión por quemadura.

\begin{tabular}{|c|c|c|}
\hline Nutriente & Dosis diaria sugerida & Comentario \\
\hline Lípidos & $1.0-1.5 \mathrm{~g} / \mathrm{kg} / \mathrm{d}$ & $<30 \%$ de las calorías no proteicas \\
\hline \multirow{2}{*}{ Hidratos de carbono } & & Aporte no debe exceder $5.0 \mathrm{mg} / \mathrm{kg} / \mathrm{min}$ manteniendo \\
\hline & & Glucemia 140-180 mg/dL con insulina IV (en ausencia de diabetes mellitus) \\
\hline Glutamina & $0.3-0.5 \mathrm{~g} / \mathrm{kg} / \mathrm{d}$ & Vía enteral exclusiva. No administrar en presencia de disfunción hepática y renal \\
\hline Zinc & $25-40 \mathrm{mg}$ & \\
\hline Vitamina C & $1.0-3.0 \mathrm{~g}$ & En las primeras 24 horas se ha recomendado $66 \mathrm{mg} / \mathrm{kg} / \mathrm{h}$ en el manejo del estado de choque \\
\hline Vitamina D & $\begin{array}{l}\leq 70 \text { años: } 600 \mathrm{UI} \\
>70 \text { años: } 800 \mathrm{UI}\end{array}$ & Vitamina D3 (oral, enteral o parenteral). No hay una recomendación definitiva de suplementación \\
\hline Vitamina A & $10.000 \mathrm{UI}$ & \\
\hline Vitamina $\mathrm{E}$ & 20-25 UI & \\
\hline
\end{tabular}

$\mathrm{UI}=$ unidades internacionales.

Los elementos traza tales como zinc, selenio y cobre juegan un rol como antioxidantes endógenos de defensa en el organismo. Los pacientes quemados críticos se caracterizan por un aumento en la producción de radicales libres y una consecuente depleción en el balance de elementos traza. Una consideración importante ha sido la suplementación de zinc en el paciente quemado, puesto que participa en la adecuada cicatrización de heridas, en la función linfocitaria y en la síntesis proteica. Sin embargo, pese a esto, no hay evidencia basada en las guías de práctica clínica que avale la reposición de elementos traza ${ }^{16}$ (Tabla 2).

\section{Terapia farmacológica}

En los últimos años se han investigado distintos fármacos para reducir el catabolismo o mejorar el anabolismo del paciente quemado. Los fármacos más importantes utilizados en este sentido incluyen hormonas anabólicas (hormona de crecimiento, insulina, IGF-1, combinaciones de IGF-1 e IGFBP-3, oxandrolona y testosterona), aunque su uso sigue siendo controversial (Tabla 3).

El factor de crecimiento similar a la insulina tipo 1 (IGF-I) es un péptido que muestra una gran semejanza con la proinsulina, que además tiene propiedades metabólicas y anabólicas muy similares a la insulina. Se ha observado que ayuda a mantener la integridad de la mucosa intestinal, mantener la función inmune y atenuar la respuesta inflamatoria observada en los pacientes quemados. ${ }^{17}$

La hormona de crecimiento humano recombinante administrada intramuscular de manera diaria durante la hospitalización por quemaduras ha mostrado múltiples efectos sobre el metabolismo y la inflamación postquemadura. Tiene profundos efectos anabólicos en los músculos y la piel, por efectos directos a través del receptor de hormona de crecimiento o por efectos indirectos a través del factor de crecimiento similar a la insulina. ${ }^{18}$ Parece ser una opción de tratamiento ideal para el hipermetabolismo en quemaduras; sin embargo, Takala y colaboradores encontraron que altas dosis de rhGH se asociaron con un aumento sustancial de la morbilidad y la mortalidad, por lo que su uso fue restringido. ${ }^{19}$

La oxandrolona es un análogo de la testosterona que en pacientes quemados mejora el catabolismo proteico muscular a través de un aumento de la síntesis proteica, reduce la pérdida de peso y aumenta la cicatrización en el sitio de herida, inclusive el tratamiento a largo plazo con oxandrolona disminuye el hipermetabolismo crónicamente elevado, aumenta la masa corporal magra y el contenido mineral óseo. Sin embargo, se ha observado también que los pacientes dependientes de ventilación mecánica que recibían oxandrolona tenían un curso más prolongado de ventilación asistida y cursaban con síndrome de insuficiencia respiratoria aguda, además de que la oxandrolona puede causar daño hepático agudo.

\section{Propranolol}

El uso del propranolol se ha propuesto en pacientes quemados por diversos motivos. Por un lado, las catecolaminas endógenas son mediadores primarios de la respuesta hipermetabólica a las quemaduras, esta respuesta sistémica se caracteriza por el desarrollo de una circulación hiperdinámica, un aumento del gasto energético basal y del catabolismo de la proteína muscular. El bloqueo del estímulo beta-adrenérgico disminuye la termogénesis suprafisiológica, el trabajo cardiaco y el gasto energético en reposo. Además, el propranolol mejora el reciclaje de los aminoácidos libres intracelulares, lo que aumenta la eficiencia de la síntesis pro- 
Tabla 3: Agentes anabólicos.

\begin{tabular}{lll}
\hline Agente anabólico & Sitio de acción & Efecto \\
\hline Insulina & Hígado & Disminuye la proteólisis \\
& Musculoesquelético & Mejora la masa corporal magra \\
& Tejido graso & Disminuye la oxidación lipídica \\
Propranolol & Tejido graso & Disminuye lipólisis periférica \\
& Hígado & Promueve la homeostasis de la glucosa \\
& Catecolaminas & Disminuye la activación de genes involucrados en la gluconeogénesis \\
Factor de crecimiento & Musculoesquelético & Bloqueo no selectivo de los receptores B-adrenérgicos \\
& & Promueve la homeostasis de la glucosa similar a la insulina tipo 1 \\
Hormona de crecimiento & Musculoesquelético & Disminuye la proteólisis \\
Oxandrolona & Musculoesquelético & Múltiples efectos anabólicos \\
& & Disminuye la proteólisis \\
\hline
\end{tabular}

teica en el músculo, disminuye la lipólisis periférica e incrementa la eficiencia del hígado en secretar ácidos grasos libres. Incluso el tratamiento a largo plazo redujo significativamente el aumento persistente de la frecuencia cardiaca y el gasto energético en reposo, evitó la pérdida ósea y mejoró la masa corporal magra. El bloqueo beta-adrenérgico con propranolol representa probablemente la terapia anticatabólica (bloqueando el receptor de catecolamina) más eficaz en el tratamiento de las quemaduras. ${ }^{20}$

\section{Rehabilitación}

La pérdida prolongada de musculoesquelético y la inmovilización forzosa dejan a las víctimas de quemaduras caquécticas y con poca condición física. La restauración de la masa muscular y la función es un componente esencial de la rehabilitación de los sobrevivientes de quemaduras. Se ha demostrado que el entrenamiento con ejercicios de rehabilitación activa es seguro y eficaz, iniciados de manera temprana e incluso durante la ventilación mecánica, en términos de restaurar la masa corporal magra, la condición cardiorrespiratoria y la fuerza muscular en los sobrevivientes de quemaduras. ${ }^{1}$

\section{CONCLUSIONES}

Una quemadura severa es una lesión extremadamente devastadora que afecta a casi todos los sistemas y órganos, que conduce a una morbilidad y mortalidad significativa, y se acompaña de una respuesta hipermetabólica característica. El estado hipermetabólico prolongado del paciente quemado se vuelve perjudicial y se asocia con un vasto catabolismo, fallo multiorgánico y muerte. Este aumento del hipermetabolismo es responsable del alto catabolismo que se observa en las quemaduras graves con glucólisis acelerada, proteólisis y lipólisis que conducen a la pérdida de peso y la erosión de la masa corporal magra, fatiga generalizada y una respuesta inmune debilitada.
Se han ideado varias estrategias terapéuticas para mejorar la respuesta hipermetabólica, incluido el control ambiental, la escisión de tejido lesionado y muerto para reducir la estimulación inflamatoria, el soporte nutricional y las terapias farmacológicas. Se requiere una dieta alta en proteínas y alta en calorías suplementada con multivitaminas y oligoelementos para los pacientes quemados. La ruta de alimentación también ha demostrado ser importante.

En las últimas décadas, se han probado varias intervenciones farmacológicas en pacientes con quemaduras, principalmente para intentar reducir la respuesta hipermetabólica.

\section{REFERENCIAS}

1. Porter C, Tompkins RG, Finnerty CC, Sidossis SL, Suman EO, Herndon ND. The metabolic stress response to burn trauma: current understanding and therapies. Lancet. 2016;388:14171426.

2. Jeschke MG. Post-burn hypermetabolism: past, present and future. J Burn Care Res. 2016;37:86-96.

3. Fagan SP, Bilodeau ML. Burn intensive care. J Burn Care Res. 2016;37:86-96.

4. Bilodeau PA, Coyne ES, Wing SS. The ubiquitin proteasome system in atrophying skeletal muscle: roles and regulation. $A m \mathrm{~J}$ Physiol Cell Physiol. 2016;311:392-403.

5. Zamudio AJ, Peña RM, Riesgo EJ. La ubiquitinación: un sistema de regulación dinámico de los organismos. Rev Esp Cienc QuímBiol. 2012;15:133-141.

6. Cree MG, Wolfe RR. Postburn trauma insulin resistance and fat metabolism. Am J Physiol Endocrinol Metab. 2008;209:1-9.

7. Kraft R, Herndon DN, Finnerty CC, Hiyama Y, Jeschke MG. Association of postburn fatty acids and triglycerides with clinical outcome in severely burned children. $J$ Clin Endocrinol Metab. 2013;98:314-321.

8. Rehou S, Mason S, Burnett M, Jeschke M. Burned adults develop profound glucose intolerance. Crit Care Med. 2016;44:1059-1066.

9. Gore DC, Chinkes DL, Hart DW, Wolf SE, Herndon DN, Sanford AP. Hyperglycemia exacerbates muscle protein catabolism in burn-injured patients. Crit Care Med. 2002;30:2438-3442.

10. Randle PJ, Garland PB, Hales CN, Newsholme EA. The glucose fatty-acid cycle. Its role in insulin sensitivity and the metabolic disturbances of diabetes mellitus. Lancet. 1963;1:785-789.

11. Moreiraa E, Burghiby G, Manzanares W. Metabolismo y terapia nutricional en el paciente quemado crítico: una revisión actualizada. Med Intensiva. 2018;42(5):306-316. www.elsevier. es/medintensiva 
12. Evidence-Based Guidelines Group ABA. Practice guidelines for burn care. J Burn Care Rehabil. 2001;22:59-66.

13. García de Lorenzo y Mateos A, Ortiz Leyba C, Sánchez Sánchez SM. Recomendaciones para el soporte nutricional y metabólico especializado del paciente crítico. Actualización. Consenso SEMICYUC-SENPE: paciente quemado crítico. Med Intensiva.2011;35:63-67.

14. Rimdeika R, Gudaviciene D, Adamonis K, Barauskas G, Pavalkis $D$, Endzinas Z. The effectiveness of caloric value of enteral nutrition in patients with major burns. Burns. 2006;32:83-86.

15. Dickerson RN, Gervasio JM, Riley ML, et al. Accuracy of predictive methods to estimate resting energy expenditure of thermally injured patients. JPENJ Parenter Enteral Nutr. 2002;26:17-29.

16. Abdullahi A, Jeschke MG. Nutrition and anabolic pharmacotherapies in the care of burn patients. american society for parenteral and enteral nutrition. Nutr Clin Pract. 2014;29(5):621-630.
17. Jeschke MG, Barrow RE, Herndon DN. Insulin like growth factor I plus insulin like growth factor binding protein 3 attenuates the proinflammatory acute phase response in severely burned children. Ann Surg. 2000;231:246-252.

18. Jeschke MG, Herndon DN, Wolf SE, et al. Recombinant human growth hormone alters acute phase reactant proteins, cytokine expression, and liver morphology in burned rats. J Surg Res. 1999;83:122-129.

19. Takala J, Ruokonen E, Webster NR, et al. Increased mortality associated with growth hormone treatment in critically ill adults. N Engl J Med. 1999;341:785-792.

20. Núñez VT, Sánchez M, Millán P, García LA. Revisión sistemática del efecto del propranolol sobre el hipermetabolismo del quemado. Med Intensiva. 2015;39:101-113.

Correspondencia:

Marco Antonio Garnica Escamilla

E-mail: teranestmarco@yahoo.com.mx 